\title{
Spin Dynamics in Hybrid Iron Oxide-Gold Nanostructures
}

\author{
T. Orlando, ${ }^{1, *}$ A. Capozzi, ${ }^{2,}$ E. Umut,${ }^{3,4}$ L. Bordonali, ${ }^{1}$ M. Mariani,${ }^{5}$ P. Galinetto, ${ }^{1}$ F. Pineider, ${ }^{6}$ P. Masala, ${ }^{7}$ \\ F. Tabak, ${ }^{3}$ M. Scavini, ${ }^{7,8}$ P. Santini, ${ }^{9}$ M. Corti, ${ }^{1}$ C. Sangregorio, ${ }^{6,}{ }^{10}$ P. Ghigna, ${ }^{11}$ and A. Lascialfari ${ }^{12,13}$ \\ ${ }^{1}$ Department of Physics, Università di Pavia, and Consorzio INSTM, Pavia, I-27100, Italy \\ ${ }^{2}$ Institute of Physics of Biological Systems, EPFL, Lausanne, CH-1015, Switzerland \\ ${ }^{3}$ Physics Engineering Department, Hacettepe University, Ankara, Turkey \\ ${ }^{4}$ School of Medical Sciences, Department of Medical Imaging Techniques, Dokuz Eylul University, Izmir, Turkey \\ ${ }^{5}$ Department of Physics and Astronomy, Università di Bologna, and Consorzio INSTM, Bologna, I-40127, Italy \\ ${ }^{6}$ Department of Chemistry, Università di Firenze, \\ and Consorzio INSTM, Sesto Fiorentino, I-50019, Italy \\ ${ }^{7}$ Department of Chemistry, Università degli Studi di Milano, and Consorzio INSTM, Milano, I-20133, Italy \\ ${ }^{8}$ CNR-ISTM, Milano, I-20133, Italy \\ ${ }^{9}$ Dipartimento di Fisica e Scienze della Terra, Università degli Studi di Parma, Parma, I-43124, Italy \\ ${ }^{10}$ CNR-ICCOM, Sesto Fiorentino, I-50019, Italy \\ ${ }^{11}$ Department of Chemistry, Università di Pavia, and Consorzio INSTM, Pavia, I-27100, Italy \\ ${ }^{12}$ Department of Physics, Università degli Studi di Milano, and Consorzio INSTM, Milano, I-20133, Italy \\ ${ }^{13}$ Istituto di Nanoscienze, CNR, Modena, I-41125, Italy
}

\begin{abstract}
We report a broadband ${ }^{1} \mathrm{H}-\mathrm{NMR}$ study of the spin dynamics of coated maghemite and goldmaghemite hybrid nanostructures with two different geometries, namely dimers and coreshells. All the samples have a superparamagnetic behaviour, displaying a blocking temperature $T_{B} \sim 80 \mathrm{~K}$ (maghemite), $\sim 105 \mathrm{~K}$ (dimer), $\sim 150 \mathrm{~K}$ (core-shell) and the magnetization reversal time follows the Vogel-Fulcher law. We observed three different anomalies in ${ }^{1} \mathrm{H}-\mathrm{NMR} T_{1}^{-1}$ vs. $T$ that decrease in amplitude when increasing the applied magnetic field. We suggest that the anomalies are related to three distinct system dynamics: molecular rotations of the organic groups $(240<T<270 \mathrm{~K})$, superparamagnetic spin blockage $(100<T<150 \mathrm{~K})$ and surface-core spins dynamics $(T<25$ $\mathrm{K})$. By fitting the $T_{1}^{-1}$ data with a heuristic model, we achieved a good agreement with magnetic relaxation data and literature values for methyl-organic rotation frequencies.
\end{abstract}

\section{INTRODUCTION}

In the last 30 years much effort has been devoted to the development of novel advanced nanostructured materials based on a proper combination of magneto-optically active and plasmonic inorganic components tailored at the nanoscale ${ }^{1-3}$. From an applicative standpoint, this new class of multifunctional magneto-plasmonic nanoheterostructures offers the detection capability of plasmonic nanostructures, used as sensors in optics, photonics and biomedicine ${ }^{4,5}$, combined with the potential of magnetic nanoparticles in medical diagnostics, therapeutics and sensing ${ }^{6,7}$. The study of such hybrid nanostrucures involves some fundamental issues about the interplay between plasmonic and magnetic properties. Specifically, the influence of the plasmonic resonance on the magneto-optical properties of the system and the ability of the magnetic field, in turn, to modulate the plasmonic resonance are nowadays quite widely investigated ${ }^{8}$, both theoretically $^{9,10}$ and experimentally ${ }^{11}$.

In this work we focus our attention on the spin dynamics of magneto-plasmonic nanostructures, constituted by maghemite $\left(\gamma-\mathrm{Fe}_{2} \mathrm{O}_{3}\right)$, playing the role of the magnetic component, and gold as the plasmonic component. In order to investigate the influence of the metal on the surface spin dynamics at a microscopic level, we opted for two different geometries, i.e. dimers and coreshells: in dimers, a spherical maghemite nanoparticle and a gold one are bound together with a single contact interface; in coreshells, a gold spherical core is sorrounded by a maghemite shell.

A pure spherical shaped maghemite sample was also studied as a reference. All samples are coated with oleylamine in order to minimize interparticle interactions. After some standard chemico-physical characterization (Raman spectroscopy, X-ray diffraction and magnetometry), ${ }^{1} \mathrm{H}-\mathrm{NMR}$ spin-lattice nuclear relaxation times and spectra as a function of temperature were measured in order to gain access to the local dynamic properties of the magnetic system. As will be argued, these properties emerge as a consequence of a number of physical phenomena, namely molecular rotation, reversal of the superparamagnetic moment, and surface spin dynamics. Using a heuristic model based on the original theoretical results by Moriya on nuclear relaxation in paramagnets ${ }^{12,13}$, we were able to properly fit the $T>100 \mathrm{~K}$ data, quantifying the correlation times and the activation energy barriers for two distinct physical mechanisms.

\section{SAMPLES AND EXPERIMENTAL TECHNIQUES}

Samples of maghemite $\gamma-\mathrm{Fe}_{2} \mathrm{O}_{3}(\mathrm{MG}), \gamma-\mathrm{Fe}_{2} \mathrm{O}_{3}-\mathrm{Au}$ dimers (DM) and $\gamma-\mathrm{Fe}_{2} \mathrm{O}_{3}$-Au coreshells (CS) have been synthesized in powder form, as described in Ref. [14]. MG particles were prepared heating up a mixture of $\mathrm{Fe}(\mathrm{acac})_{3}, 1,2$-hexadecanediol and oleic acid under a flow of nitrogen ${ }^{15}$. DM sample were synthesized using decom- 
TABLE I. Summary of the properties of the magneto-plasmonic nanostructures investigated: $d$ is the mean diameter of the whole nanostructure (iron-oxide plus gold) core obtained from TEM measurements, while $d_{m a g}$ is the diameter of the magnetic part (NB:for CS $d_{m a g}$ is the thickness of the maghemite shell); $T_{B}$ is the blocking temperature at $H=50$ Oe; $\tau_{0}^{a c}$ is the attempt time of the Arrhenius-like formula for the superparamagnetic correlation time, $\tau_{c, S P M}$, while $E_{b}^{a c}$ is the activation energy barrier. Both $\tau_{0}^{a c}$ and $E_{b}^{a c}$ are estimated from ac susceptibility data using a Vogel-Fulcher law, where the offset parameter is $T_{0}$. For further details, please refer to Ref. [14].

\begin{tabular}{|c|c|c|c|c|c|c|c|}
\hline Sample & label & $d(\mathrm{~nm})$ & $d_{m a g}(\mathrm{~nm})$ & $T_{B}(\mathrm{~K})$ & $\tau_{0}^{a c}(\mathrm{~s})$ & $E_{b}^{a c}(\mathrm{~K})$ & $T_{0}(\mathrm{~K})$ \\
\hline$\gamma-\mathrm{Fe}_{2} \mathrm{O}_{3}$ & MG & $7.5 \pm 1.0$ & $7.5 \pm 1.0$ & $80 \pm 10$ & $9.80 \cdot 10^{-10}$ & 572 & 0.01 \\
\hline $\mathrm{Au}-\gamma-\mathrm{Fe}_{2} \mathrm{O}_{3}$ dimer & $\mathrm{DM}$ & $11.0 \pm 1.0$ & $7.1 \pm 1.0$ & $105 \pm 20$ & $1.37 \cdot 10^{-9}$ & 797 & 40.9 \\
\hline $\mathrm{Au}-\gamma-\mathrm{Fe}_{2} \mathrm{O}_{3}$ coreshell & $\mathrm{CS}$ & $15.9 \pm 0.3$ & $4.7 \pm 1.3$ & $150 \pm 20$ & $8.90 \cdot 10^{-9}$ & 1173 & 53.5 \\
\hline
\end{tabular}

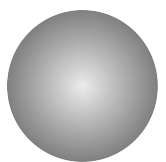

maghemite

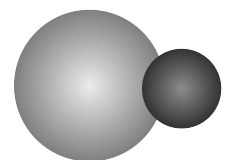

dimer

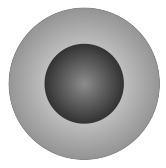

coreshell
FIG. 1. Qualitative sketches (not to scale) of the examined samples: maghemite is drawn in light grey, whereas gold in dark grey.

position of $\mathrm{Fe}(\mathrm{CO})_{5}$ on the surface of Au nanoparticles ${ }^{16}$. Finally, to prepare CS, the iron oxide was nucleated on previously synthesized gold seeds ${ }^{17}$. An illustrative sketch of the geometries considered for this work is shown in FIG. 1. All the samples are coated with oleylamine.

Micro-Raman measurements were carried out at room temperature with a Labram Dilor spectrometer equipped with an Olympus microscope HS BX40. The $632.8 \mathrm{~nm}$ light from He-Ne laser was employed as excitation source. The samples, mounted on a motorized $x y$ stage, were tested with a $100 \mathrm{x}$ objective and with a laser spot of $\sim 0.7 \mu \mathrm{m}$ of diameter. The attained spectral resolution was about $1 \mathrm{~cm}^{-1}$. Neutral filters with different optical density were applied to attenuate light intensity, leading to power density values ranging from $5 \times 102 \mathrm{~W} / \mathrm{cm}^{2}$ to $5 \times 105 \mathrm{~W} / \mathrm{cm}^{2}$. To avoid undesirable thermal effects, the power density and acquisition times were kept to the lowest feasible level; this is the reason for the low quality signal/noise ratio of this set of measurements. Sample phase homogeneity has been verified by mapping the Raman spectra from different regions of the samples.

XRPD patterns were collected at the ID31 beamline of the ESRF facility in Grenoble ${ }^{18}$ by collecting data at room temperature, under incident wavelength $\lambda=$ $0.40000(2) \AA$. The samples were loaded in $0.5 \mathrm{~mm}$ glass capillaries and then allowed to spin to reduce preferred orientations. $\mathrm{A} \mathrm{CeO}_{2}$ standard sample was employed to calibrate the instrumental line profile broadening parameters. The wavelength was selected by means of a double-crystal $\mathrm{Si}(111)$ monochromator. The diffracted intensities were detected through nine $\mathrm{Si}(111)$ analyzer crystals, which covered $16^{\circ}$ in $2 \theta$. The average crystallographic structure was determined applying the Rietveld method ${ }^{19}$, using the software GSAS $^{20}$ with the graphical interface EXPGUI ${ }^{21}$. The line profile and the background were fitted with pseudo-Voigt functions and Chebyschev polynomials, respectively. The data were corrected using $f^{\prime}$ and $f^{\prime \prime}$ to take into account the anomalous scattering ${ }^{22}$. Cell parameters and isotropic mean square displacement $(m s d)$ parameters were varied.

Magnetic dc susceptibility measurements were performed on a MPMS-XL7 Quantum Design superconducting quantum interference device (SQUID) magnetometer in the temperature range $2-300 \mathrm{~K}$ at various applied magnetic fields, varying from 50 Oe to 16600 Oe.

By probing ${ }^{1} \mathrm{H}$ nuclei in oleylamine coating, nuclear spin-lattice relaxation times, $T_{1}$, and spin-spin relaxation times, $T_{2}$, were measured using a standard pulsed NMR spectrometer in the temperature range $[1.7 \mathrm{~K}, 300 \mathrm{~K}]$ and at two different applied magnetic fields, i.e. $\mathrm{H}=6.5 \mathrm{kOe}$, $16.5 \mathrm{kOe}$, corresponding, respectively, to $27.7 \mathrm{MHz}$ and $70.2 \mathrm{MHz}$ for the proton Larmor frequency. All the samples were investigated as dry powders. The NMR signal was sampled using standard solid echo sequences $\left(90^{\circ}\right.$ $\tau_{\text {echo }}-90^{\circ}$ ) for $T_{2}$ measurements, preceded by a comb of $90^{\circ}$ pulses, saturating the NMR line, for $T_{1}$ measurements.

The recovery of the longitudinal nuclear magnetization was found to be non exponential at both Larmor frequencies, i.e. $\nu=27.7 \mathrm{MHz}, 70.2 \mathrm{MHz}$. A deviation from the mono-exponential behavior can be related to a distribution of nuclear relaxation rates due to the presence of inequivalent proton sites and a powder distribution. Therefore, in order to measure a consistent relaxation parameter, an effective $T_{1}$ reflecting the fastest relaxing nuclei, defined as the time at which the nuclear magnetization has recovered $40 \%$ of the equilibrium value, was taken into account.

\section{RAMAN SPECTROSCOPY}

The spectrum from DM sample in the region 100-1800 $\mathrm{cm}^{-1}$ is reported in FIG. 2(a). The Raman modes characteristic for iron oxides are observed within the 100-1000 $\mathrm{cm}^{-1}$ range ${ }^{23-27}$. Although it is well known that the frequency of maghemites Raman active phonon modes can vary with the preparation method and distribution of vacancies within the maghemite crystal unit cell ${ }^{23}$, the 
maghemite Raman spectrum is generally composed of three Raman active phonon modes at $365 \mathrm{~cm}^{-1}\left(T_{2 g}\right)$, $511 \mathrm{~cm}^{-1}\left(E_{g}\right)$, and $700 \mathrm{~cm}^{-1}\left(A_{1 g}\right)$. The latter is the most intense and exhibit a broad scattering response $\mathrm{e}^{23,25}$. Concerning linewidhts and energy positions, maghemite and magnetite display similar but dinstinguishable Raman spectra. Indeed, the $A_{1 g}$ mode in FIG. 2(a) for DM sample is consistent with a predominant maghemite fraction, even though the presence of a lower amount of magnetite phase cannot be excluded.

The magnetite spectrum is peaked at $670 \mathrm{~cm}^{-1}$ and markedly narrower. Its spectral features are also characterized by a weak response, indicating poor scattering properties typical for maghemite structure, further reduced by the small size of scattering nanoparticles. The sharp and intense lines observed in the region above 1000 $\mathrm{cm}^{-1}$ are mainly due to vibrational units involving carbon atoms from the organic capping agent.

Some information can be inferred comparing spectra from DM, CS and MG samples. The Raman spectra for these samples in the region $100-800 \mathrm{~cm}^{-1}$ are reported in FIG. 2(b). At first we notice that in the spectrum from the starting material the Raman signal at around $700 \mathrm{~cm}^{-1}$ is characterized by two bands peaked approximately at $670 \mathrm{~cm}^{-1}$ and $705 \mathrm{~cm}^{-1}$, the first band having a higher intensity. This two-component feature smeared out in CS and DM samples, which exhibit broadened spectral features with a maximum around $690 \mathrm{~cm}^{-1}$. This behaviour could be ascribed to a slightly higher amount of magnetite phase in the starting material, with a consequent partial conversion during the formation of nano-aggregates. The spectral broadening is also consistent with a greater disorder for $\mathrm{AuNps} @ \mathrm{Fe}_{x} \mathrm{O}_{y}$ particles. Higher disorder degree causes a weakening of selection rules with a structureless scattering in the whole Raman region, in particular for CS sample.

An indirect confirmation of a higher degree of disorder is provided by the analyses of Raman lines in both samples, once the phase transformation to the hematite phase has been induced by laser heating. By the bestfitting procedure on the so-obtained $\mathrm{A}_{1} g$ mode at about $215 \mathrm{~cm}^{-1}$ we derived the values for the FMHM for both samples, i.e. $9.50 \mathrm{~cm}^{-1}$ and $20.40 \mathrm{~cm}^{-1}$ for DM and CS respectively. According to [27] the first value is consistent with an average particle size close to $7 \mathrm{~nm}$, and thus in agreement with [14]. The value for CS indicates a lower average size of nanoparticles and a greater surface/volume ratio due to the coreshell morphology, leading to a higher disorder degree.

A net difference in the spectra reported in FIG. 2(b) is observed at lower wavenumbers. For the DM sample an intense band peaks at about $250 \mathrm{~cm}^{-1}$. On the other hand, the $250 \mathrm{~cm}^{-1}$ feature is dominant in the Raman spectra of lepidocrocite ${ }^{23}$, which is an iron hydroxide with an orthorhombic crystal structure: $\gamma$-FeOOH is a common product of iron oxidation and it is paramagnetic at room temperature and antiferromagnetic below $50 \mathrm{~K}^{28}$. If $\gamma-\mathrm{FeOOH}$ is in the proximity of gold nanoparti-
TABLE II. Rietveld refinement parameters for DM and CS samples. Au phases belong to $F \mathrm{~d}-3 m$ space group, while $\gamma$ $\mathrm{Fe}_{2} \mathrm{O}_{3}$ ones to $P 4_{3} 32$. WF is the weight fraction.

\begin{tabular}{lcc}
\hline \hline Parameter & Dimer $(\mathrm{DM})$ & Coreshell $(\mathrm{CS})$ \\
$\mathrm{a} / \AA \mathrm{Au}_{\text {broad }}$ & $4.0672(4)$ & $4.0687(5)$ \\
$\mathrm{a} / \AA \mathrm{Au}_{\text {sharp }}$ & $4.0770(5)$ & $4.0802(6)$ \\
$\% \mathrm{WF} \mathrm{Au}$ broad & $23.5(6)$ & $27.7(1)$ \\
$\% \mathrm{WF} \mathrm{Au} \mathrm{Au}_{\text {broad }}$ & $46.1(5)$ & $1.2(2)$ \\
$\mathrm{a} / \AA \gamma-\mathrm{Fe}_{2} \mathrm{O}_{3}$ & $8.356(2)$ & $8.3564(8)$ \\
$\% \mathrm{WF} \gamma-\mathrm{Fe}_{2} \mathrm{O}_{3}$ & $30.4(6)$ & $71.1(1)$ \\
$\mathrm{U} / \AA^{2}$ & $0.0026(3)$ & $0.0046(3)$ \\
$\mathrm{Rp}$ & 0.074 & 0.065 \\
$\mathrm{R}\left(\mathrm{F}^{2}\right)$ & 0.038 & 0.050 \\
\hline \hline
\end{tabular}

cles, the Raman scattering can be enhanced by the SERS effect. Nevertheless, we cannot rule out for this signal an active role of other SERS mechanisms, due to bonds involving atoms from the capping agent. The absence of this signal in the CS sample could be justified by the different conformational arrangement of nano-aggregates. The localized nature of bonds in the DM sample is reflected by a high SERS response.

\section{XRPD MEASUREMENTS}

The experimental patterns of (a) DM and (b) CS, together with their best fits, are reported in FIG. 3. The refined parameters are reported in TABLE II. These patterns were described using two $\mathrm{Au}$ phases, space group $F$ d-3m, and a $\gamma-\mathrm{Fe}_{2} \mathrm{O}_{3}$ maghemite phases, space group $\mathrm{P}_{3} 32$. The nanostructure of the samples is evidenced by the broadness of the diffraction peaks. It should be noted that, thanks to the high $Q$ resolution and to the good counting statistics obtained at the ID31 beamline, we are able to distinguish between maghemite $\left(\gamma-\mathrm{Fe}_{2} \mathrm{O}_{3}\right.$, space group $\mathrm{P}_{3} 32$ for the disordered phase and space group $P 4_{1} 2_{1} 2$ for the ordered one) and magnetite $\left(\mathrm{Fe}_{3} \mathrm{O}_{4}\right.$, space group $F \mathrm{~d}-3 m$ ). Indeed, magnetite and maghemite differ in cell parameter $\left(\mathrm{a}=8.344 \AA\right.$ for maghemite ${ }^{29}$ and $\mathrm{a}=8.397 \AA$ for magnetite ${ }^{30}$ ) and in the presence of superstructure peaks in maghemite due to the different extinction rules of the two space groups, even in the disordered case. These superstructure peaks are evident in CS, while they are not detectable in DM, probably due to the larger weight phase fraction of gold. However, the cell parameter is an univocal marker of the nature of the iron oxide phase $^{31}$. Our Rietveld refinements then suggest the presence of maghemite rather than magnetite. As shown in TABLE II the atomic means square displacement (U) for CS is almost twice the DM one. Since the data were collected at the same $\mathrm{T}$, this is a qualitative indication of increased disorder in CS sample with respect to8 DM, in agreement with Raman findings.

Concerning the gold phases, the shape of the diffrac- 


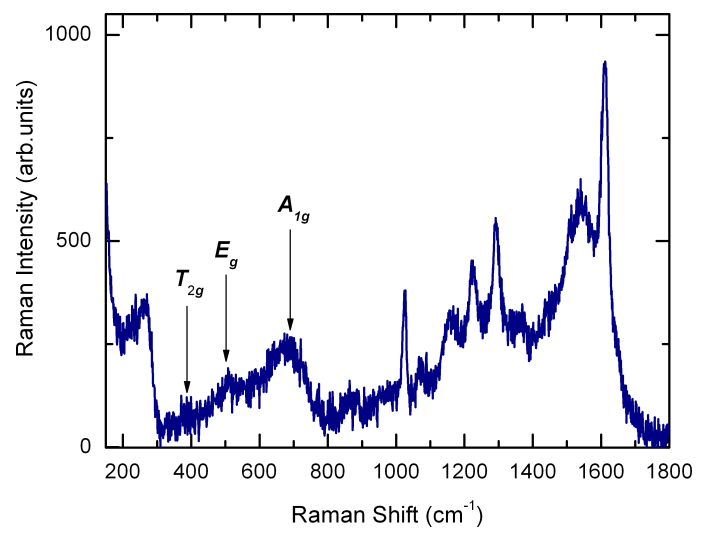

(a)

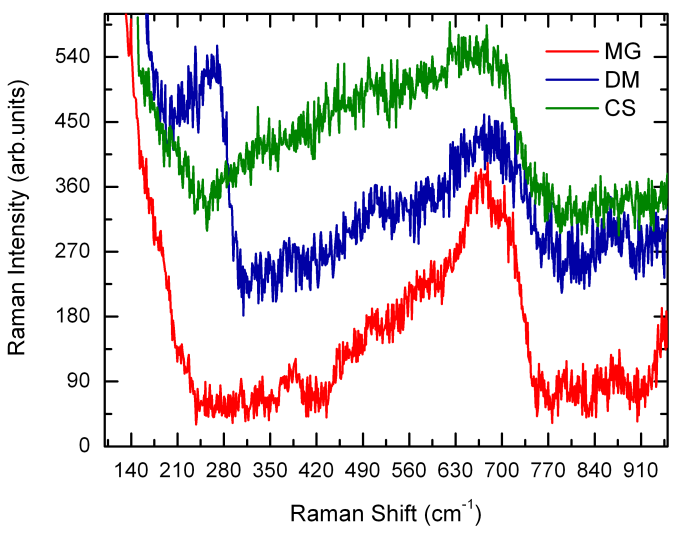

(b)

FIG. 2. (a) Raman spectrum of the DM sample: the arrows indicate the Raman active modes for maghemite, i.e. $T_{2 g}, E_{g}$ and $A_{1 g}$. (b) Raman spectrum comparison fo MG, DM and CS in the wavelenght range $100 \div 800 \mathrm{~cm}^{-1}$.

tion peaks suggests that in both DM and CS a bimodal distribution is present. For Rietveld refinement we fitted the pertinent peaks using two gold phases, both coming from space group $\mathrm{Fd}-3 m$, labeled as $\mathrm{Au}_{\text {broad }}$ and $\mathrm{Au}_{\text {sharp }}$ (FIG. 3(b) inset), because they are characterized by broad and sharp peaks, respectively. As suggested by previously performed TEM inspections ${ }^{14}$, sharp peaks could be related to the aggregation of $\mathrm{Au}$ into clusters, while broad peaks are related to gold nanoparticles tightly bound to maghemite. In the latter, due to its small particle size, we observed the shrink of $\mathrm{Au}$ cell parameter with respect to Au aggregate and to bulk $(\mathrm{a}=4.078 \AA)$, as reported in literature ${ }^{32,33}$.

\section{MAGNETIC MEASUREMENTS}

The investigated nanostructures, as expected for ironoxide fine particles under a critical diameter $d_{C}$ of the order of $10 \div 100 \mathrm{~nm}$, exhibit a superparamagnetic behaviour and an uniaxial magnetic anisotropy: consequently, the total magnetic moment $m$ of the single nanoparticle in the ground state can assume two different orientations, i.e. one parallel and one antiparallel to the anisotropy axis. An energy barrier $E_{b}$ separates the two degenerate ground state energy levels.
The magnetic moment reversal time between these two orientation is known as the Néel relaxation time, $\tau_{N}$, which obeys an activated Arrhenius-like law, i.e. $\tau_{N}=$ $\tau_{0} \exp \left(E_{b} /\left(k_{B} T\right)\right)$, assuming the absence of interparticle interaction. When interparticle dipolar interaction can not be neglected, as is often the case of powder samples, a "glassy state" may emerge ${ }^{34,35}$. Consequently, $\tau_{N}$ assumes the well-known Vogel-Fulcher form: $\tau_{N}=$ $\tau_{0} \exp \left(E_{b} / k_{B}\left(T-T_{0}\right)\right)$. Without an applied static magnetic field, the superparamagnetic energy barrier is proportional to the nanoparticle volume, i.e. $E_{b}=K_{\text {eff }} V$, where $K_{\text {eff }}$ is the effective anisotropy constant ${ }^{36}$. The size distribution of these nanostructures is, as previously demonstrated $^{14}$, a log-normal curve; thus, in the following sections we will adopt the same anisotropy energy barrier distribution, i.e.:

$$
\rho\left(E_{b}\right)=\frac{1}{E_{b} \sqrt{2 \pi \sigma^{2}}} e^{-\frac{\ln E_{b}-\mu}{2 \sigma^{2}}}
$$

A quantitative estimate of parameters $\mu$ and $\sigma^{2}$, mean and the variance of the distribution, respectively, can be obtained by fitting the ZFC magnetization curve at low field ( $H=50$ Oe) using the following formula ${ }^{37,38}$ :

$$
M_{Z F C}=\frac{\mu H M_{S}^{2} k_{B}}{3 k_{e f f}^{2}}\left(\frac{1}{T} \int_{0}^{E_{l i m}} E_{b}^{2} \rho\left(E_{b}\right) d E_{b}+\int_{E_{l i m}}^{\infty} E_{b}^{2} \rho\left(E_{b}\right) d E_{b}\right)
$$

where parameter $E_{\text {lim }}$ depends on the temperature. The fitting results are shown in TABLE III and, using Eq. 1, the estimated energy barrier distribution can be drawn for each sample (FIG. 4). It is evident that the presence of gold and the complex sample morphology correlates with an increase of the anisotropy energy barrier. In particular, the energy barrier distribution for $\mathrm{DM}$ is quite similar to the one of the spherical maghemite 


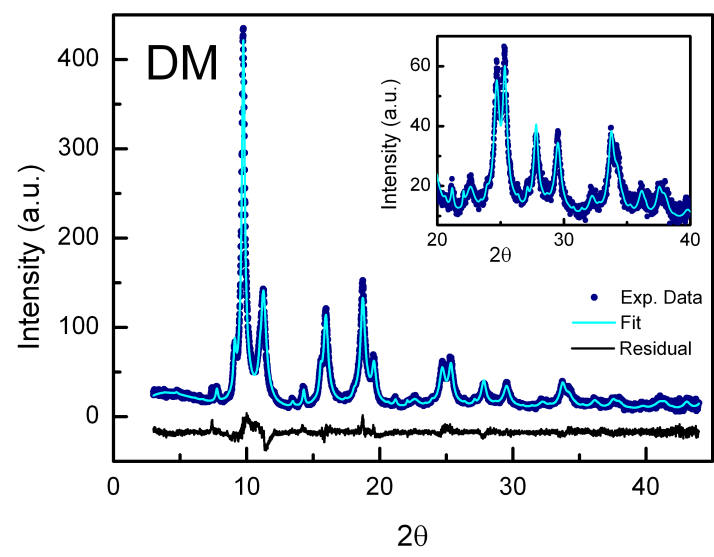

(a)

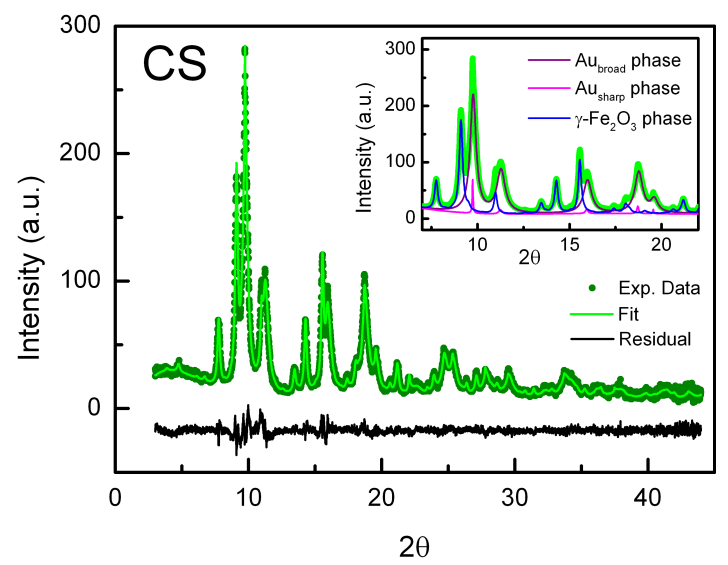

(b)

FIG. 3. Refinements for (a) DM and (b) CS: experimental pattern, calculated and residual. The inset in (a) reports Rietveld refinement at large angles to highlight the high quality of data. In the inset of (b) the calculated pattern for each of the three phases in CS are shown: total fit (green line), $\gamma-\mathrm{Fe}_{2} \mathrm{O}_{3}$ phase, $\mathrm{Au}_{\text {broad }}$ phase and $\mathrm{Au}_{\text {sharp }}$ phase.

nanoparticle, i.e. MG: this means that the gold nanoparticle attached to the maghemite surface is responsible for only a small increase of magnetic anisotropy, a contribution likely ascribed to the local rearrangement of the surface spins in proximity of the contact region. Conversely, the CS nanostructure exhibit a significant enhancement of anisotropy energy barrier, probably due to the complex arrangement of spins on both the inner and outer surface of the maghemite shell. Using the mean values $E_{b}$ obtained for MG, DM and CS, the effective anisotropy constants $K_{\text {eff }}$ for the investigated nanostructures can be calculated. Recalling that both surface and core anisotropy contribute to the total effective anisotropy of the system $^{36,39}$, i.e. $K_{\text {eff }}=(6 / d) \cdot K_{\text {surf }}+K_{\text {core }}$, the results shown in TABLE III can be commented as follows. The $K_{\text {eff }}$ estimate for MG is in agreement with the values reported in literature ${ }^{40}$ for spherical maghemite nanoparticles with a mean diameter of $7 \mathrm{~nm}$; the DM sample features a greater effective anisotropy constant
TABLE III. Log-normal parameters, $\mu$ and $\sigma$, obtained fitting ZFC data at $H=50$ Oe with Eq. 2. $E_{b}$ and $\delta E$ are the energy mean value and its standard deviation, respectively. The effective anisotropy constant is calculated using the relationship $E_{b}=K_{e f f} V$, where $V$ is the maghemite volume.

\begin{tabular}{cccccc}
\hline \hline Sample & $\mu$ & $\sigma$ & $E_{B}(\mathrm{~K})$ & $\delta E$ & $K_{\text {eff }}\left(\mathrm{erg} / \mathrm{cm}^{3}\right)$ \\
MG & 5.54 & 0.77 & 341.4 & 305.3 & $2.14 \cdot 10^{5}$ \\
DM & 5.86 & 0.86 & 508.8 & 532.7 & $3.75 \cdot 10^{5}$ \\
CS & 6.59 & 0.78 & 989.4 & 901.4 & $0.69 \cdot 10^{5}$ \\
\hline \hline
\end{tabular}

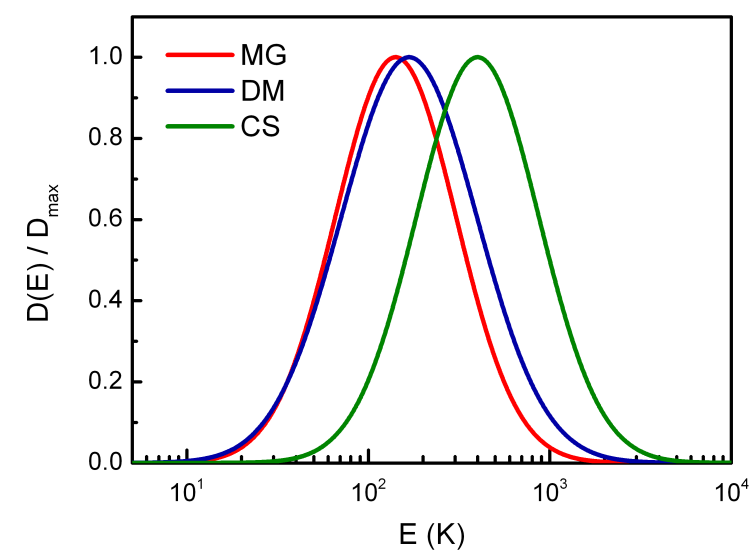

FIG. 4. Energy barrier distributions for MG, DM and CS calculated by fitting the ZFC curve at $H=50$ Oe with Eq. 2: $\mu$ and $\sigma$ values are reported in TABLE III.

than the $\mathrm{MG}$ sample, i.e. $K_{\text {eff }}^{D M}>K_{\text {eff }}^{M G}$, possibly due to the presence of the gold nanoparticle, which increases the surface anisotropy contribution. On the other hand, $K_{e f f}^{C S}$ is one order of magnitude lower with respect to MG and DM: this difference can be interpreted as a direct consequence of the nanoparticle heterostructure, namely the lack of a central magnetic region leads to a lower core anisotropy (the core is $\mathrm{Au}$ and the shell is maghemite).

Assume now to apply an external magnetic field that dampens the magnetic anisotropy barrier ${ }^{41}$ : consequently, the peak in the ZFC curves (FIG. 5), marking the so-called superparamagnetic blocking temperature $\left(T_{B} \propto E_{b}\right)$, shifts towards a lower temperature when the applied magnetic field increases, and eventually disappears for high fields $(H>5.5 \mathrm{kOe})$. The evolution of the superparamagnetic blocking temperature as a function of the field is described by a power law, i.e. $H^{2 / 3} \sim\left(1-T_{B} / T_{0}\right)$, where $T_{0}$ is the blocking temperature at zero field ${ }^{42}$. This relation, known as the Almeida-Thouless line, is typical of spin glasses, but can also hold true for superparamagnetic systems ${ }^{43}$, under the assumption $m H \ll E_{b}$, where $m$ is the total magnetic moment of the single nanoparticle; on the other hand, when $m H \sim E_{b}$ the model is no longer valid and a disagreement between the experiment and theoretical predictions can be observed (FIG. 6). The crossover field, i.e. the field that marks the separation between 


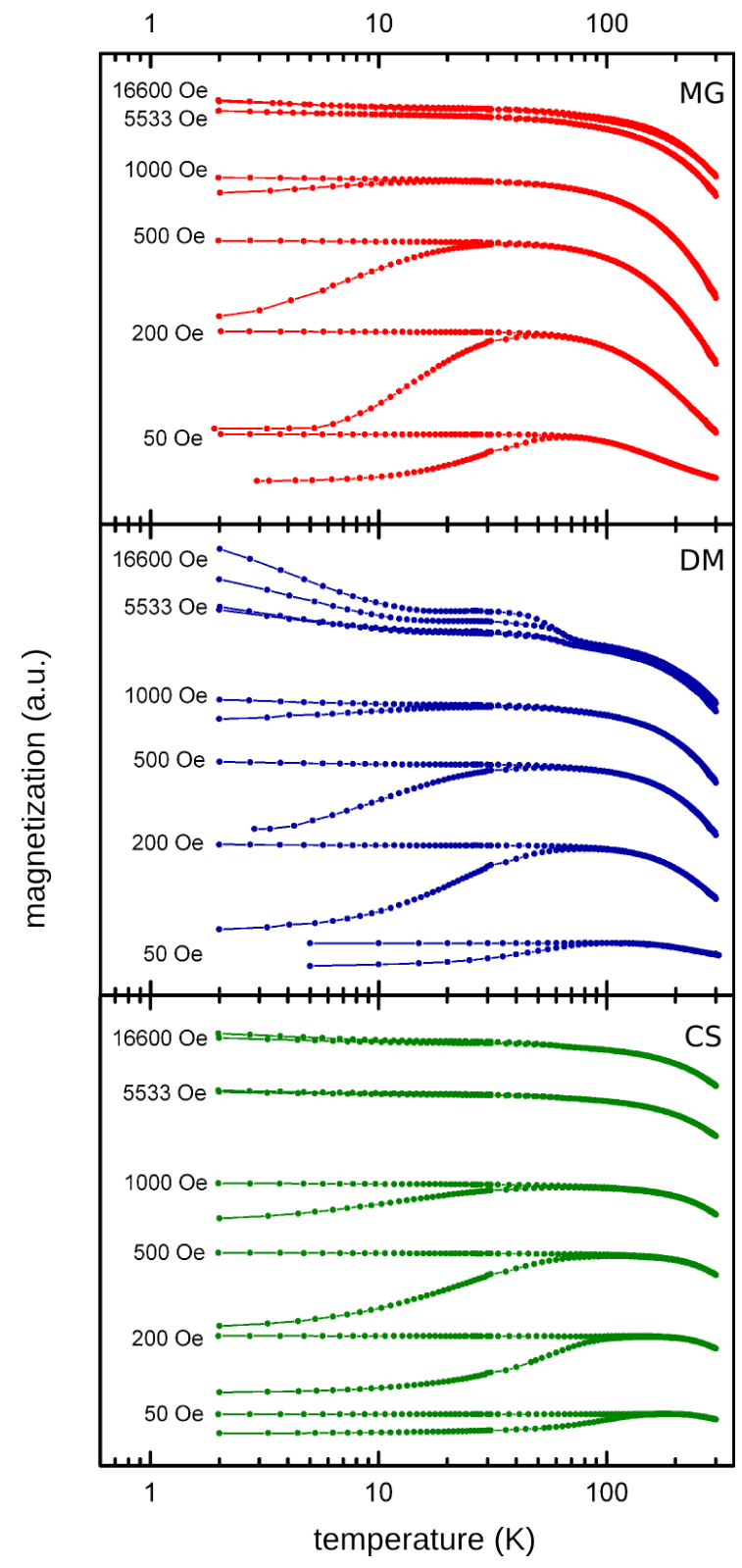

FIG. 5. ZFC/FC magnetization curves (arbitrary units) for six different applied magnetic fields as a function of temperature for maghemite (top), dimers (middle) and coreshells (bottom). The evolution of $T_{B}$, i.e. the temperature at which the maximum of ZFC curve occurs, is easy to follow. Paramagnetic impurities affect DM measurements and their contribution is visible at low temperatures $(T<10 \mathrm{~K})$ for $H>5$ $\mathrm{kOe}$.

the Almeida-Thouless regime and non-linear high field regime, is $H_{C} \sim 525$ Oe for all our samples. For weakly interacting fine particles ${ }^{44} H_{C}$ is ca. 150 Oe. Thus, the value of 525 Oe is compatible with the powder form of our samples, where interparticles interactions can not be neglected.

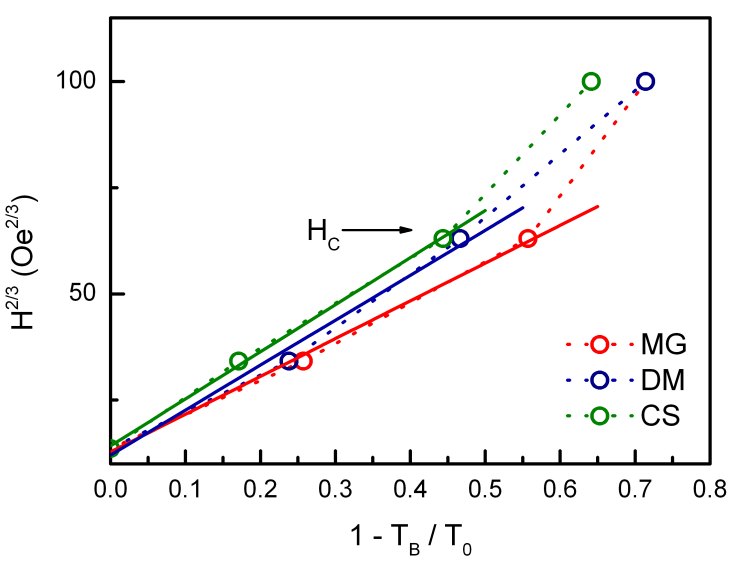

FIG. 6. Evolution of the blocking temperature $T_{B}$ as a function of the applied magnetic field. $H_{C} \sim 525$ Oe marks the crossover field between the Almeida-Thouless regime and non-linear high field regime. The parameter $T_{0}$ was set equal to $T_{B}$ at 50 Oe.

TABLE IV. Temperature corresponding to $T_{1}^{-1}$ maxima: the $h, i$ and $l$ subscripts mark, respectively, the high, the intermediate and the low peak.

\begin{tabular}{ccccc}
\hline \hline Sample & Frequency $(\mathrm{MHz})$ & $T_{h}(\mathrm{~K})$ & $T_{i}(\mathrm{~K})$ & $T_{l}(\mathrm{~K})$ \\
\multirow{2}{*}{$\mathrm{MG}$} & 27.7 & $275(12)$ & $120(6)$ & $11(2)$ \\
& 70.2 & $278(12)$ & $135(7)$ & $30(5)$ \\
$\mathrm{DM}$ & 27.7 & $244(5)$ & $130(5)$ & $13(3)$ \\
& 70.2 & $252(3)$ & $140(5)$ & $20(4)$ \\
$\mathrm{CS}$ & 27.7 & $259(10)$ & $139(10)$ & $5(1)$ \\
& 70.2 & $270(7)$ & $142(10)$ & $15(3)$ \\
\hline \hline
\end{tabular}

\section{NMR SPECTRA AND RELAXATION RATES}

The experimental data sets of $T_{1}^{-1}$ as a function of temperature for MG, DM and CS are presented in Figure 7. As one can notice, the behaviour of the longitudinal relaxation rate is qualitatively independent from the nanostructure composition and morphology, except for the low temperature region $(T<30 \mathrm{~K})$. Indeed, over a "background" slowly increasing with temperature, three different anomalies can be identified, in correspondence of three different temperatures. Furthermore, when the static magnetic field increases, besides the damping of the relaxation rate, a shift of the position of the peaks towards higher temperature can be observed (TABLE IV).

As already proven in literature, the observation of a peak in $T_{1}^{-1}(T)$ curve is related to the existence of electronic spin fluctuations, whose consequence is the fast nuclear relaxation of the system via a convenient energy exchange channel between the electronic system and the nuclear system. As a matter of fact, if $\tau_{c}$ is the characteristic correlation time of a specific dynamic motion (of molecules, spins etc.), the Bloembergen-Purcell-Pound 

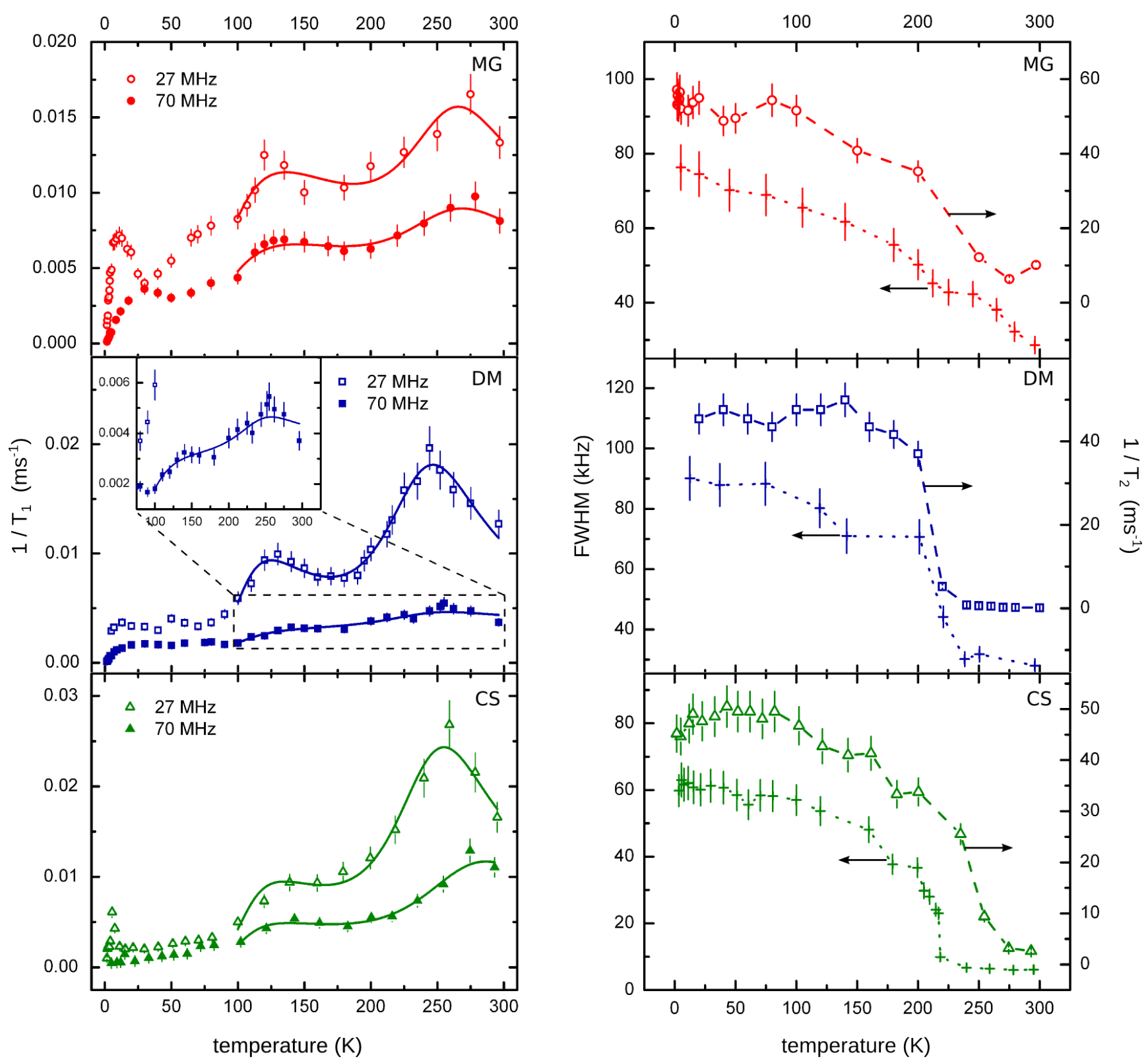

FIG. 7. (left) Longitudinal relaxation time $1 / T_{1}$ plotted as a function of $T$ for the three nanostructures examined in this work: maghemite NPs (top), dimers (middle) and coreshells (bottom). In addition to the experimental data points, the best fit curves to Eq. 3 are shown. (right) Transverse relaxation time $1 / T_{2}$ and full width at half maximum (FWHM) as a function of $T$, collected at $\nu=27.7 \mathrm{MHz}, H=6.5 \mathrm{kOe}$ for each sample.

(BPP) theory ${ }^{45}$ predicts that $1 / T_{1}$ should peak when $\tau_{c} \omega_{L} \approx 1$, where $\omega_{L}$ is the proton Larmor frequency.

According to these experimental observations we hypothesized that the three anomalies could be due to three different physical mechanisms. First of all, the existence of molecular groups containing hydrogen nuclei allowed us to relate the high temperature peak to molecular rotational motions; in particular the rotations of $\mathrm{CH}_{2}$ and/or $\mathrm{CH}_{3}$ groups in the organic coating of the nanoparticles are assumed to be responsible for such an anomaly, occurring when the inverse of the rotational correlation time becomes of the order of $\omega_{L}$ (i.e. $\tau_{\text {c.rot }} \omega_{L} \approx 1$ ). Furthermore, these motions produce a narrowing of the NMR spectral line of protons for $T>200 \mathrm{~K}$, as reported in FIG. 7 ; the transverse relaxation rate $1 / T_{2}$ follows the behaviour of the FWHM, being $T_{2}$ proportional to $1 / \delta \omega$, where $\delta \omega$ is the line width.

The peak at intermediate temperature (i.e. within the range $70<T<180 \mathrm{~K}$ ) is ascribed to the fulfillment of the condition $\omega_{L} \tau_{N} \approx 1$, where $\tau_{N}$ is the correlation time of the superparamagnetic reorientation of the magnetization inside the maghemite nanoparticle (MG, DM) or the 
TABLE V. Results of the best fit procedure of longitudinal nuclear relaxation rate, $1 / T_{1}$, with Eq. 3. Attempt correlation times $\left(\tau_{0, S P M}\right.$ and $\left.\tau_{0, r o t}\right)$ and energy barriers $\left(E_{b, S P M}\right.$ and $\left.E_{b, r o t}\right)$ are shown. The standard error obtained from the algorithm is given in brackets.

\begin{tabular}{cccccc}
\hline \hline Sample & Frequency $(\mathrm{MHz})$ & $\tau_{0, S P M}(\mathrm{~s})$ & $E_{b, S P M} / k_{B}(\mathrm{~K})$ & $\tau_{0, \text { rot }}(\mathrm{s})$ & $E_{b, \text { rot }} / k_{B}(\mathrm{~K})$ \\
$\mathrm{MG}$ & 27.7 & $3.45(1.26) \cdot 10^{-10}$ & $326(51)$ & $1.93(0.21) \cdot 10^{-12}$ & $2141(47)$ \\
& 70.2 & $1.74(0.08) \cdot 10^{-10}$ & $299(10)$ & $1.94(0.20) \cdot 10^{-12}$ & $1913(30)$ \\
$\mathrm{DM}$ & 27.7 & $3.15(0.51) \cdot 10^{-10}$ & $224(14)$ & $2.02(0.13) \cdot 10^{-12}$ & $1969(136)$ \\
& 70.2 & $5.25(0.75) \cdot 10^{-10}$ & $126(15)$ & $1.90(0.45) \cdot 10^{-12}$ & $1797(60)$ \\
$\mathrm{CS}$ & 27.7 & $5.93(0.57) \cdot 10^{-10}$ & $160(7)$ & $1.78(0.40) \cdot 10^{-12}$ & $2061(50)$ \\
& 70.2 & $2.98(0.46) \cdot 10^{-10}$ & $141(12)$ & $1.97(0.31) \cdot 10^{-12}$ & $2016(38)$ \\
\hline \hline
\end{tabular}

shell $(\mathrm{CS})^{12}$.

Finally, the low temperature peak is possibly connected to a more complex spin dynamics inside the maghemite part, in particular to a contribution to the dynamics coming from the surface spins, which are able to just partially sense the Néel-like dynamics of the particle core. On the other hand, the latter contribution to $T_{1}^{-1}$ could be strongly influenced by the proximity of the gold component to the maghemite and by the morphology of the nanoparticle as a whole. Indeed, taking into account that the contact surface among $\mathrm{Au}$ and $\gamma-\mathrm{Fe}_{2} \mathrm{O}_{3}$ is more extended in the coreshell structure, it can be argued that: (i) the peak occurring in fine $\mathrm{MG}$ is about two times higher (at the lowest field) than the "background"value of $T_{1}^{-1}$; this observation allows us to hypothesize that this peak reflects the pure maghemite surface spins contribution; (ii) on the contrary, in the dimer sample (DM), the peak is almost quenched for both fields and only a broad shoulder is visible; this could be due to the lack of crystallinity in the maghemite component, as pointed outby Raman spectrosopy investigation; (iii) in the core-shell sample, the peak reaches a value three times higher than the "background" which allows more spins at the surface of magnetic core to enter in contact with gold (at the lowest field), an occurrence probably due to the morphology and to the $\mathrm{Au}-\gamma-\mathrm{Fe}_{2} \mathrm{O}_{3}$ interactions. As a final remark, we should observe that the anomalies at $T<30 \mathrm{~K}$ follow the same qualitative behaviour as the intermediate and the high temperature ones, i.e. decreasing in value and displacing towards higher temperatures as the magnetic field increases.

To obtain quantitative information about the spin dynamics of these magneto-plasmonic nanostructures, we performed an analysis of the experimental data employing well-known heuristic models: a simple Bloembergen Purcell Pound (BPP)-like spectral density function ${ }^{45}$ can be used for all the observed correlation times, i.e.: $J(\omega, T) \propto \tau_{c} /\left(1+\tau_{c} \omega_{L}^{2}\right)$, where $\tau_{c}$ is the correlation time. To account for the rotational motion correlation time $\tau_{\text {rot }}$ we chose an activated behaviour as a function of temperature, following the Arrhenius law well accepted in literature: $\tau_{c, \text { rot }}=\tau_{0, \text { rot }} \exp \left(\frac{E_{b, \text { rot }}}{k_{B} T}\right)$, where $E_{b, \text { rot }}$ is the energy barrier and $\tau_{0, r o t}$ is the attempt rotational correlation time. For the intermediate temperature anomaly, attributed to the maghemite magnetization reversal time, we assumed the existence of dipolar interparticle interactions ${ }^{36}$ that lead to a superparamagnetic correlation time that follows the Vogel-Fulcher expression, i.e. $\tau_{c, S P M}=\tau_{0, S P M} \exp \left(\frac{E_{b, S P M}}{k_{B} \cdot\left(T-T_{0}\right)}\right)$, where $E_{b, S P M}$ is the energy barrier, $\tau_{0}$ is the attempt time and $k_{B} T_{0}$ is the interparticle interaction energy. This assumption is supported by the ac susceptibility investigation ${ }^{14}$, which are sensitive to the spin magnetization blockage and allow to evaluate the attempt time $\tau_{0, S P M}$ (the results are reported in TABLE I). Due to the complexity of the phenomenon and the lack of previous investigations, the low temperature peak was not considered in our analysis.

The data for $T>100 \mathrm{~K}$ were fitted to the following equation:

$$
\frac{1}{T_{1}}=A \chi(T) T \cdot \frac{\tau_{c, S P M}}{1+\tau_{c, S P M} \omega_{L}^{2}}+B \cdot \frac{\tau_{c, r o t}}{1+\tau_{c, r o t} \omega_{L}^{2}}
$$

where $A$ and $B$ are arbitrary constants proportional to the square of the hyperfine fields fluctuations at the proton nuclear sites. The factor $\chi(T) T$ represents the contribution to the nuclear relaxation rate arising from the static magnetic properties and its value corresponds to the effective magnetic moments of the nanoparticle. In the fitting procedure, $T_{0}$ was fixed to the best fit values obtained from the analysis of ac susceptibility data (TABLE I).

Equation 3 depends upon four free parameters, i.e. the energy barriers $\left(E_{b, r o t}\right.$ and $\left.E_{b, S P M}\right)$ and the correlation times $\left(\tau_{c, \text { rot }}\right.$ and $\left.\tau_{c, S P M}\right)$. For a consistent data fitting, we enforced a few constraints: (i) the rotational correlation time constant, $\tau_{c, r o t}$, and the related barrier, $E_{b, r o t}$, were constrained to a small range enclosing the values reported in literature and (ii) the superparamagnetic reorientation correlation time, $\tau_{c, S P M}$ was bound to be not more of an order of magnitude larger or smaller than the one obtained from magnetometry measurements (i.e. ac susceptibility).

The curves resulting from the best fit procedure are shown in FIG. 7, while the parameters are collected in TABLE V. The values of $\tau_{0, \text { rot }}$ and $E_{b, \text { rot }}$ are mostly field- 
independent and there does not appear to be any relationship with the nanostructure morphology, in agreement with the rotational barrier not being dependent on the external magnetic field in presence of the same coating (i.e. oleylamine). Additionally, their values are close to the ones found in literature ${ }^{46,47}$. On the other hand, the $\tau_{0, S P M}$ values are compatible with the ones obtained from ac susceptibility (TABLE I) and those reported in literature for $\gamma-\mathrm{Fe}_{2} \mathrm{O}_{3}$ nanoparticles of similar diameter ${ }^{48,49}$. As expected, a strong suppression of the superparamagnetic barrier $E_{b, S P M}$ with an increase in the applied magnetic field is observed.

Discrepancies between the values of $E_{b}$ estimated with AC susceptibility and NMR (see TABLE I and V) can be reconciled by recalling how the activation energy barrier is affected by the application of a magnetic field ${ }^{41}$ : first of all, the average barrier in the particle ensemble is naturally damped by a polarizing applied field, which introduces a bias between the two possible orientation of the magnetization along the principal anisotropy axis; secondly, since AC susceptibility measurements are performed at very low fields (10 Oe in our case $\left.{ }^{14}\right)$, the contribution to the total anisotropy coming from surface and collective phenomena is expected to be decidedly heavier with respect to when a higher magnetic field, suitable for NMR experiments, is applied. Indeed, even a moderately high magnetic field, of the order of $10^{2} \mathrm{Oe}$, is capable of completely quenching any collective/disordered phase ${ }^{50}$ (i.e. a spin-glass-like phase or a superspin-glass phase), in agreement with the observations in Sec. V.

An additional evidence that corroborates this hypothesis is provided by the dependence of $E_{b}$ on topology in both NMR and AC susceptibility measurements: analysis of AC susceptibility data yields differences between $E_{b}$ values of about $600 \mathrm{~K}$ between $\mathrm{MG}$ and $\mathrm{CS}$, and about $400 \mathrm{~K}$ between DM and CS; if, on the other hand, we inspect $E_{b}$ in the case of NMR at the highest applied field $H=16.5 \mathrm{kOe}$, we observe much lower differences between MG and DM (173 K) and between MG and CS $(158 \mathrm{~K})$. We thus deduce that those contributions to the effective anisotropy coming from interparticle interactions and surface coordination, which are active at zero applied fields, play little to no role in defining $E_{b}$ when a field is applied.

\section{CONCLUSIONS}

A complete magnetic characterization, Raman spectroscopy, XRPD and broadband ${ }^{1} \mathrm{H}-\mathrm{NMR}$ investigation of two different magneto-plasmonic nanostructures were presented. We studied hybrid samples $\mathrm{Au}-\left(\gamma-\mathrm{Fe}_{2} \mathrm{O}_{3}\right)$ with two different morphologies, i.e dimers and coreshells, using pure maghemite $7 \mathrm{~nm}$ spherical nanoparticles as a reference sample, in order to have a better understanding of the influence of gold and of the hybrid geometry on the magnetic properties and spin dynamics. High resolution XRPD unambiguously determined the presence of nano-structured maghemite in both samples, and the shrinking of Au cell parameter in the Au- $\gamma$ $\mathrm{Fe}_{2} \mathrm{O}_{3}$ nanocomposite with respect to the bulk. SQUID ac susceptibility measurements showed the increase of the magnetic anisotropy energy barrier $E_{b}$ when gold is bound to the maghemite: possibly, the metal nanoparticle perturbs the surface spin arrangement, resulting in an increased surface anisotropy. Furthermore, even if the coreshell sample exhibits a higher $E_{b}$ with respect to dimer one, we observe that $K_{e f f}^{C S}<K_{e f f}^{D M}$, as a consequence of a lower bulk magnetic anisotropy in the CS geometry. ${ }^{1} \mathrm{H}-\mathrm{NMR}$ allowed us to study the spin dynamics in the three different nanostructures. Three anomalies in the longitudinal nuclear relaxation rate have been observed, which were related to distinct dynamics: rotational motions of organic groups, superparamagnetic reorientation of the magnetization, and, probably, surface spin dynamics. A simple BPP model was successfully used to fit the intermediate and high temperature data, i.e. $T>100 \mathrm{~K}$, obtaining a correct estimate of the correlation times and of the energy barriers, as proved by the agreement with the literature values evaluations from magnetometry results. A spin dynamics possibly related to complex surface-core spin interactions was observed in the low temperature region, but a theoretical explanation of this phenomenon is still missing in literature; thus, future more detailed investigation is planned.

\section{ACKNOWLEDGMENTS}

The research was coordinated under the project Fondazione Cariplo no. 20100612. FIRB project RINAME no. RBAP114AMK and INSTM-Regione Lombardia MAG-NANO are also acknowledged.

\footnotetext{
* T.O. and A.C. contributed equally to this work. Correspondence should be addressed to: tomas.orlando@unipv.it

${ }^{1}$ D. Jamon, F. Donatini, a. Siblini, F. Royer, R. Perzynski, V. Cabuil, and S. Neveu, Journal of Magnetism and Magnetic Materials 321, 1148 (2009).
}

${ }^{2}$ F. Royer, D. Jamon, J.-J. Rousseau, H. Roux, D. Zins, and V. Cabuil, Applied Physics Letters 86, 011107 (2005).

3 J. B. Gonzlez-Daz, A. Garca-Martn, J. M. Garca-Martn, A. Cebollada, G. Armelles, B. Seplveda, Y. Alaverdyan, and M. Kll, Small 4, 202 (2008). 
${ }^{4}$ M. E. Stewart, C. R. Anderton, L. B. Thompson, J. Maria, S. K. Gray, J. a. Rogers, and R. G. Nuzzo, Chemical Review 108, 494 (2008).

5 T. Chung, S.-Y. Lee, E. Y. Song, H. Chun, and B. Lee, Sensors 11, 10907 (2011).

${ }^{6}$ Q. Pankhurst, J. Connolly, and S. Jones, Journal of Physics D: Applied Physics 36, R167 (2003).

7 Q. Pankhurst, N. Thanh, S. Jones, and J. Dobson, Journal of Physics D: Applied Physics 42, 224001 (2009).

8 G. Armelles, A. Cebollada, A. Garcia-Martin, J. GarcíaMartín, M. González, J. González-Díaz, E. Ferreiro-Vila, and J. Torrado, Journal of Optics A: Pure and Applied Optics 11, 114023 (2009).

${ }^{9}$ V. V. Temnov, G. Armelles, U. Woggon, D. Guzatov, A. Cebollada, A. Garcia-Martin, J.-M. Garcia-Martin, T. Thomay, A. Leitenstorfer, and R. Bratschitsch, Nature Photonics 4, 107 (2010).

10 V. I. Belotelov, L. L. Doskolovich, and a. K. Zvezdin, Physical review letters 98, 077401 (2007).

11 P. K. Jain, Y. Xiao, R. Walsworth, and A. E. Cohen, Nano Letters 9, 1644 (2009).

${ }^{12}$ L. Bordonali, Y. Furukawa, M. Kraken, F. Litterst, C. Sangregorio, M. F. Casula, and A. Lascialfari, Physical Review B 85, 1 (2012).

13 T. Moriya, Progress of Theoretical Physics 28, 371 (1962).

14 E. Umut, F. Pineider, P. Arosio, C. Sangregorio, M. Corti, F. Tabak, A. Lascialfari, and P. Ghigna, Journal of Magnetism and Magnetic Materials 324, 2373 (2012).

15 S. Sun, H. Zeng, D. B. Robinson, S. Raoux, P. M. Rice, S. X. Wang, and G. Li, Journal of the American Chemical Society 126, 273 (2004), http://pubs.acs.org/doi/pdf/10.1021/ja0380852.

${ }^{16} \mathrm{H}$. Yu, M. Chen, P. Rice, S. Wang, and R. White, Nano Letters 5, 379 (2005).

17 W. Shi, H. Zeng, Y. Sahoo, T. Y. Ohulchanskyy, Y. Ding, Z. L. Wang, M. Swihart, and P. N. Prasad, Nano Letters 6, 875 (2006), http://pubs.acs.org/doi/pdf/10.1021/nl0600833.

18 A. Fitch, Journal of Research of the National Institute of Standards and Technology 109, 133 (2004).

19 H. M. Rietveld, Journal of Applied Crystallography 2, 65 (1969).

${ }^{20}$ R. V. Dreele and A. Larson, Los Alamos National Laboratory Report LAUR, Tech. Rep. (2004).

${ }^{21}$ B. H. Toby, Journal of Applied Crystallography 34, 210 (2001).

${ }^{22}$ S. Brennan and P. L. Cowan, Review of Scientific Instruments 63 (1992).

23 D. L. A. de Faria, S. Venncio Silva, and M. T. de Oliveira, Journal of Raman Spectroscopy 28, 873 (1997).

${ }^{24}$ R. Gupta, A. K. Sood, P. Metcalf, and J. M. Honig, Phys. Rev. B 65, 104430 (2002).

25 I. Chamritski and G. Burns, The Journal of Physical Chemistry B 109, 4965 (2005), pMID: 16863155, http://pubs.acs.org/doi/pdf/10.1021/jp048748h.

26 O. N. Shebanova and P. Lazor, Journal of Raman Spectroscopy 34, 845 (2003).

27 I. V. Chernyshova, M. F. Hochella Jr, and A. S. Madden, Phys. Chem. Chem. Phys. 9, 1736 (2007).

28 A. M. Hirt, L. Lanci, J. Dobson, P. Weidler, and A. U. Gehring, Journal of Geophysical Research: Solid Earth 107, EPM 5 (2002).

29 Z. Somogyvari, E. Svb, G. Mszros, K. Krezhov, I. Nedkov, I. Saj, and F. Boure, Applied Physics A 74, s1077 (2002).

30 D. M. Lind, S. D. Berry, G. Chern, H. Mathias, and L. R. Testardi, Phys. Rev. B 45, 1838 (1992).

31 R. Frison, G. Cernuto, A. Cervellino, O. Zaharko, G. M. Colonna, A. Guagliardi, and N. Masciocchi, Chemistry of Materials 25, 4820 (2013).

32 J. Luo, M. M. Maye, V. Petkov, N. N. Kariuki, L. Wang, P. Njoki, D. Mott, Y. Lin, and C.-J. Zhong, Chemistry of materials 17, 3086 (2005).

33 P. M. Diehm, P. Ágoston, and K. Albe, ChemPhysChem 13, 2443 (2012).

34 J. L. Dormann, R. Cherkaoui, L. Spinu, M. Nogue, F. Lucari, D. Fiorani, A. Garcia, E. Tronc, and J. P. Jolivet, Journal of Magnetism and Magnetic Materials 187, L139 (1998).

35 J. Dormann, L. Spinu, E. Tronc, J. Jolivet, F. Lucari, F. D'Orazio, and D. Fiorani, Journal of Magnetism and Magnetic Materials 183, L255 (1998).

36 O. Iglesias and A. Labarta, Physical Review B 70, 1 (2004).

37 F. Tournus and E. Bonet, Journal of Magnetism and Magnetic Materials 323, 1109 (2011).

38 F. Tournus and a. Tamion, Journal of Magnetism and Magnetic Materials 323, 1118 (2011).

39 D. Leslie-Pelecky and R. Rieke, Chemistry of Materials 4756, 1770 (1996).

40 Y. Komorida, M. Mito, H. Deguchi, S. Takagi, a. Millan, N. J. O. Silva, and F. Palacio, Applied Physics Letters 94, 202503 (2009).

41 X. Batlle and A. Labarta, Journal of Physics D: Applied Physics 35, R15 (2002).

42 G. F. Goya and M. P. Morales, Journal of Metastable and Nanocrystalline Materials 21, 673 (2004).

43 L. Wenger and J. Mydosh, Physical Review B 2, 2 (1984).

${ }^{44}$ W. Luo, S. R. Nagel, T. Rosenbaum, and R. Rosensweig, Physical Review Letters 67, 2721 (1991).

45 N. Bloembergen, E. Purcell, and R. Pound, Physical Review 73, 679 (1948).

46 A. Kumar and C. S. J. Johnson, The Journal of Chemical Physics 60, 137 (1974).

47 J. Humphreys, Polymer 25, 1227 (1984).

${ }^{48}$ Q. Chen, A. J. Rondinone, B. C. Chakoumakos, and Z. J. Zhang, Journal of Magnetism ... 194, 1 (1999).

49 D. Kim, Y. Zhang, W. Voit, and K. Rao, Journal of Magnetism and Magnetic Materials 225, 30 (2001).

50 P. Jönsson, S. Felton, P. Svedlindh, P. Nordblad, and M. Hansen, Physical Review B 64, 212402 (2001). 